\title{
An Axiomatic Approach to Personalized Ranking Systems*
}

\author{
Alon Altman and Moshe Tennenholtz \\ Faculty of Industrial Engineering and Management \\ Technion - Israel Institute of Technology \\ Haifa 32000 \\ Israel
}

March 27, 2007

\begin{abstract}
Personalized ranking systems and trust systems are an essential tool for collaboration in a multi-agent environment. In these systems, trust relations between many agents are aggregated to produce a personalized trust rating of the agents. In this paper we introduce the first extensive axiomatic study of this setting, and explore a wide array of well-known and new personalized ranking systems. We adapt several axioms (basic criteria) from the literature on global ranking systems to the context of personalized ranking systems, and fully classify the set of systems that satisfy all of these axioms. We further show that all these axioms are necessary for this result.
\end{abstract}

\section{Introduction}

Personalized ranking systems and trust systems are an essential tool for collaboration in a multi-agent environment. In these systems, agents report on their peers' performance, and these reports are aggregated to form a ranking of the agents. This ranking may be either global, where all agents see the same ranking, or personalized, where each agent is provided with her own ranking of the agents. Examples of global ranking systems include eBay's reputation system[20] and Google's PageRank[18]. Examples of personalized ranking systems include the personalized version of PageRank[14] and the MoleTrust ranking system[8]. Furthermore, trust systems which provide each agent with a set of agents he or she can trust, can be viewed as personalized ranking systems which supply a two-level ranking over the agents. Many of these systems can be easily adapted to provide a full ranking of the agents. Examples of trust systems include OpenPGP(Pretty Good Privacy)'s trust system[10], the ranking system employed by Advogato[16], and the epinions.com web of trust.

${ }^{*}$ This paper is an extended version of [3].

Dagstuhl Seminar Proceedings 07271

Computational Social Systems and the Internet

http://drops.dagstuhl.de/opus/volltexte/2007/1152 
A central challenge in the study of ranking systems, is to provide means and rigorous tools for the evaluation of these systems. This challenge equally applies to both global and personalized ranking systems. A central approach to the evaluation of such systems is the experimental approach. In the general ranking systems setting, this approach was successfully applied to Hubs\&Authorities[15] and to various other ranking systems[9]. In the trust systems setting, [17] suggests a similar experimental approach.

A more analytical approach to the evaluation of ranking systems is the axiomatic approach. In this approach, one considers basic properties, or axioms, one might require a ranking system to satisfy. Then, existing and new systems are classified according to the set of axioms they satisfy. Examples of such study in the global ranking systems literature include $[11,9,22,5,19]$. Typical results of such study are axiomatizations of particular ranking systems, or a proof that no ranking system satisfying a set of axioms exists. For example, in [2] we provide a set of axioms that are satisfied by the PageRank system and show that any global ranking system that satisfies these axioms must coincide with PageRank.

While the axiomatic approach has been extensively applied to the global ranking systems setting, no general attempt has been made to apply such an approach to the context of personalized ranking systems. In this paper, we introduce an extensive axiomatic study of the personalized ranking system setting, by adapting axioms that have been previously applied to global ranking systems[1,4]. We compare several existing personalized ranking systems in the light of these axioms, and provide novel ranking systems that satisfy various sets of axioms. Moreover, we prove a full characterization of the personalized ranking systems satisfying all suggested axioms.

We consider four basic axioms. The first axiom, self confidence, requires that an agent would be ranked at the top of his own personalized rank. The second axiom, transitivity, captures the idea that an agent preferred by more highly trusted agents, should be ranked higher than an agent preferred by less trusted agents. The third axiom, Ranked Independence of Irrelevant Alternatives, requires that under the perspective of any agent, the relative ranking of two other agents would depend only on the pairwise comparisons between the rank of the agents that prefer them. The last axiom, strong incentive compatibility, captures the idea that an agent cannot gain trust by any agent's perspective by manipulating its reported trust preference.

We fully characterize the set of ranking systems satisfying all four axioms, and show ranking systems satisfying every three of the four axioms (but not the fourth).

This paper is organized as follows. Section 2 introduces the setting of personalized ranking systems and discusses some known system. In section 3 we present our axioms, and classify the ranking systems shown according to these axioms. In section 4 we provide a full characterization of the ranking systems satisfying all of our axioms, and in section 5 we study ranking systems satisfying every three of the four axioms. Section 6 presents some concluding remarks and suggestions for future research. 


\section{Personalized Ranking Systems}

\subsection{The Setting}

Before describing our results regarding personalized ranking systems, we must first formally define what we mean by the words "personalized ranking system" in terms of graphs and linear orderings:

Definition 2.1. Let $A$ be some set. A relation $R \subseteq A \times A$ is called an ordering on $A$ if it is reflexive, transitive, and complete. Let $L(A)$ denote the set of orderings on $A$.

Notation 2.2. Let $\preceq$ be an ordering, then $\simeq$ is the equality predicate of $\preceq$, and $\prec$ is the strict order induced by $\preceq$. Formally, $a \simeq b$ if and only if $a \preceq b$ and $b \preceq a$; and $a \prec b$ if and only if $a \preceq b$ but not $b \preceq a$.

Given the above we can define what a personalized ranking system is:

Definition 2.3. Let $\mathbb{G}_{V}$ be the set of all directed graphs $G=(V, E)$ with no parallel edges, but possibly with self-loops ${ }^{1}$. A personalized ranking system $(P R S) F$ is a functional that for every finite vertex set $V$ and for every source $s \in V$ maps every graph $G \in \mathbb{G}_{V}$ to an ordering $\preceq_{G, s}^{F} \in L(V)$.

Note that our definition of a personalized ranking system considers only the ordinal ranking of the vertices and does not assign cardinal values to vertices. Also note that our definition does not assume the existence of a path from $s$ to every vertex. However, in some settings this may be considered a useful assumption. Therefore, we shall use these kind of graphs in all examples and counter-examples, but prove our results for the more general case defined above.

\subsection{Some personalized ranking systems}

We shall now give examples of some known PRSs. A basic ranking system that is at the basis of many trust systems ranks the agents based on the minimal distance of the agents from the source.

Notation 2.4. Let $G=(V, E)$ be some directed graph and $v_{1}, v_{2} \in V$ be some vertices, we will use $d_{G}\left(v_{1}, v_{2}\right)$ to denote the length of the shortest directed path in $G$ between $v_{1}$ and $v_{2}$. If no such path exists, $d_{G}\left(v_{1}, v_{2}\right) \triangleq \infty$.

Definition 2.5. The distance PRS $F_{D}$ is defined as follows: Given a graph $G=(V, E)$ and a source $s, v_{1} \preceq_{G, s}^{F_{D}} v_{2} \Leftrightarrow d_{G}\left(s, v_{1}\right) \geq d_{G}\left(s, v_{2}\right)$

Another family of PRSs can be derived from the well-known PageRank ranking system by modifying the so-called teleportation vector in the definition of PageRank[14]. These systems can be defined as follows:

Definition 2.6. Let $G=(V, E)$ be a directed graph, and assume $V=\left\{v_{1}, v_{2}, \ldots, v_{n}\right\}$. The PageRank Matrix $A_{G}$ (of dimension $n \times n$ ) is defined as:

$$
\left[A_{G}\right]_{i, j}=\left\{\begin{array}{cc}
1 /\left|S_{G}\left(v_{j}\right)\right| & \left(v_{j}, v_{i}\right) \in E \\
0 & \text { Otherwise }
\end{array}\right.
$$

\footnotetext{
${ }^{1}$ Unless otherwise noted, all our results still apply when self loops are not allowed.
} 
The Personalized PageRank procedure ranks pages according to the stationary probability distribution obtained in the limit of a random walk with a random teleportation to the source $s$ with probability $d$; this is formally defined as follows:

Definition 2.7. Let $G=(V, E)$ be some graph, and assume $V=\left\{s, v_{2}, \ldots, v_{n}\right\}$. Let $\mathbf{r}$ be the unique solution of the system $(1-d) \cdot A_{G} \cdot \mathbf{r}+d \cdot(1,0, \ldots, 0)^{T}=\mathbf{r}$. The Personalized PageRank with damping factor $d$ of a vertex $v_{i} \in V$ is defined as $P P R_{G, s}^{d}\left(v_{i}\right)=r_{i}$. The Personalized PageRank Ranking System with damping factor $d$ is a PRS that for the vertex set $V$ and source $s \in V$ maps $G$ to $\preceq_{G, s}^{P P R_{d}}$, where $\preceq_{G, s}^{P P R_{d}}$ is defined as: for all $v_{i}, v_{j} \in V: v_{i} \preceq_{G, s}^{P P R_{d}} v_{j}$ if and only if $P P R_{G, s}^{d}\left(v_{i}\right) \leq$ $P P R_{G, s}^{d}\left(v_{j}\right)$.

We now suggest a variant of the Personalized PageRank system, which, as we will later show, has more positive properties than Personalized PageRank.

Definition 2.8. Let $G=(V, E)$ be some graph and assume $V=\left\{s, v_{2}, \ldots, v_{n}\right\}$. Let $B_{G}$ be the link matrix for $G$. That is, $\left[B_{G}\right]_{i, j}=1 \Leftrightarrow(j, i) \in E$. Let $\alpha=1 / n^{2}$ and let $\mathbf{a}$ be the unique solution of the system $\alpha \cdot B_{G} \cdot \mathbf{a}+\left(1, \alpha^{n}, \ldots, \alpha^{n}\right)^{T}=\mathbf{a}$. The $\alpha$-Rank of a vertex $v_{i} \in V$ is defined as $r_{G, s}\left(v_{i}\right)=a_{i}$. The $\alpha$-Rank PRS is a PRS that for the vertex set $V$ and source $s \in V$ maps $G$ to $\preceq_{G, s}^{\alpha R}$, where $\preceq_{G, s}^{\alpha R}$ is defined as: for all $v_{i}, v_{j} \in V: v_{i} \preceq_{G, s}^{\alpha R} v_{j}$ if and only if $r_{G, s}\left(v_{i}\right) \leq r_{G, s}\left(v_{j}\right)$.

The $\alpha$-Rank system ranks the agents based on their distance from $s$, breaking ties by the summing of the trust values of the predecessors. By selecting $\alpha=1 / n^{2}$, it is ensured that a slight difference in rank of nodes closer to $s$ will be more significant than a major difference in rank of nodes further from $s$.

Additional personalized ranking systems are presented in Section 5 as part of our axiomatic study.

\section{Some Axioms}

A basic requirement of a PRS is that the source - the agent under whose perspective we define the ranking system - must be ranked strictly at the top of the trust ranking, as each agent implicitly trusts herself. We refer to this property as self confidence.

Definition 3.1. Let $F$ be a PRS. We say that $F$ satisfies self confidence if for all graphs $G=(V, E)$, for all sources $s \in V$ and for all vertices $v \in V \backslash\{s\}: v \prec_{G, s}^{F} s$.

A basic property of (global) ranking systems called strong transitivity [1,22], which requires that if an agent $a$ 's voters are ranked higher than those of agent $b$, then agent $a$ should be ranked higher than agent $b$. We adapt this notion to the personalized setting, and provide a new weaker notion of transitivity as follows:

Notation 3.2. We will use $P_{G}(v)$ and $S_{G}(v)$ to denote the predecessor set and successor set of $v$ in $G$ respectively. The subscript $G$ may be omitted when understood from context. 
Definition 3.3. Let $F$ be a PRS. We say that $F$ satisfies quasi transitivity if for all graphs $G=(V, E)$, for all sources $s \in V$ and for all vertices $v_{1}, v_{2} \in V \backslash\{s\}$ : Assume there is a 1-1 mapping $f: P\left(v_{1}\right) \mapsto P\left(v_{2}\right)$ s.t. for all $v \in P\left(v_{1}\right): v \preceq f(v)$. Then, $v_{1} \preceq v_{2}$. $F$ further satisfies strong quasi transitivity if when $P\left(v_{1}\right) \neq \emptyset$ and for all $v \in P\left(v_{1}\right): v \prec f(v)$, then $v_{1} \prec v_{2}$. $F$ further satisfies strong transitivity if when either $f$ is not onto or for some $v \in P\left(v_{1}\right): v \prec f(v)$, then $v_{1} \prec v_{2}$.

The new notion of strong quasi transitivity requires that agents with stronger matching predecessors be ranked at least as strong as agents with weaker predecessors, but requires a strict preference only when all matching predecessors are strictly stronger.

A standard assumption in social choice settings is that an agent's relative rank should only depend on (some property of) their immediate predecessors. Such axioms are usually called independence of irrelevant alternatives(IIA) axioms. In the global ranking systems setting[1], we required that the relative ranking of two agents must only depend on the pairwise comparisons of the ranks of their predecessors, and not on their identity or cardinal value. The ranked IIA axiom differs from the one suggested by [7] in the fact that ranked IIA does not consider the identity of the voters, but rather their relative rank. We now adapt this axiom of ranked IIA to the setting of PRSs, by requiring this independence for all vertices except the source.

To formally define this condition, one must consider all possibilities of comparing two nodes in a graph based only on ordinal comparisons of their predecessors. These possibilities are called comparison profiles:

Definition 3.4. A comparison profile is a $\langle\mathbf{a}, \mathbf{b}\rangle$ where $\mathbf{a}=\left(a_{1}, \ldots, a_{n}\right), \mathbf{b}=\left(b_{1}, \ldots, b_{m}\right)$, $a_{1}, \ldots, a_{n}, b_{1}, \ldots, b_{m} \in \mathbb{N}, a_{1} \leq a_{2} \leq \cdots \leq a_{n}$, and $b_{1} \leq b_{2} \leq \cdots \leq b_{m}$. Let $\mathcal{P}$ be the set of all such profiles.

A PRS $F$, a graph $G=(V, E)$, a source $s \in V$, and a pair of vertices $v_{1}, v_{2} \in V$ are said to satisfy such a comparison profile $\langle\mathbf{a}, \mathbf{b}\rangle$ if there exist 1-1 mappings $f_{1}$ : $P\left(v_{1}\right) \mapsto\{1 \ldots n\}$ and $f_{2}: P\left(v_{2}\right) \mapsto\{1 \ldots m\}$ such that given $f:\left(\{1\} \times P\left(v_{1}\right)\right) \cup$ $\left(\{2\} \times P\left(v_{2}\right)\right) \mapsto \mathbb{N}$ defined as:

$$
\begin{gathered}
f(1, v)=a_{f_{1}(v)} \\
f(2, u)=b_{f_{2}(u)} \\
f(i, x) \leq f(j, y) \Leftrightarrow x \preceq_{G, s}^{F} y \text { for all }(i, x),(j, y) \in\left(\{1\} \times P\left(v_{1}\right)\right) \cup\left(\{2\} \times P\left(v_{2}\right)\right) .
\end{gathered}
$$

We now require that for every such profile the personalized ranking system ranks the nodes consistently:

Notation 3.5. We will use $V_{s}^{G}$ to denote the set of vertices that have a directed path from $s$ in a graph $G$. We will sloppily use $V_{s}$ when $G$ is understood from context.

Definition 3.6. Let $F$ be a PRS. We say that $F$ satisfies ranked independence of irrelevant alternatives (RIIA) if there exists a mapping $f: \mathcal{P} \mapsto\{0,1\}$ such that for every graph $G=(V, E)$, for every source $s \in V$ and for every pair of vertices $v_{1}, v_{2} \in V_{s}^{G} \backslash\{s\}$ and for every comparison profile $p \in \mathcal{P}$ that $v_{1}$ and $v_{2}$ satisfy, $v_{1} \preceq_{G, s}^{F} v_{2} \Leftrightarrow f(p)=1$. We will sloppily use the notation $\mathbf{a} \preccurlyeq \mathbf{b}$ to denote $f\langle\mathbf{a}, \mathbf{b}\rangle=1$.

This IIA axiom intuitively means that the relative ranking of agents must be consistent across all comparisons with the same rank relations. 


\subsection{Incentive Compatibility}

The issue of incentives has been extensively studied both in classical social choice[13, $21,12]$, and with regard to global ranking systems[4, 5]. As with global ranking systems, agents ranked by personalized ranking systems may wish to manipulate their reported preferences in order to improve their trustworthiness in the eyes of a specific agent. Therefore, the incentives of these agents should in many cases be taken into consideration.

We would like our ranking systems to stand against various types of manipulations. It is important to formally define what a manipuation is, and the types of manipulations we would like to defend against.

Definition 3.7. A manipulation is a function $\mathcal{M}$ that maps every graph $G=(V, E) \in$ $\mathbb{G}$ and every vertex $v \in V$ in that graph to a set of graphs $M \subseteq \mathbb{G}$ such that $G \in M$ and $v \in G^{\prime}$ for all $G^{\prime} \in M$.

That is, a manipulation defines for every vertex in any graph, what different graphs can that agent cause to be presented to the ranking system as a result of a manipulation.

Our standard of incentive compatibility is strong incentive compatibility, which requires that agents will not improve their rank in the terms of the number of agents ranked above them and the number or agents ranked the same as them ${ }^{2}$ :

Definition 3.8. Let $F$ be a PRS. $F$ satisfies strong incentive compatibility under manipulation $\mathcal{M}$ if for all true preference graphs $G=(V, E)$, for all sources $s \in V$, for all vertices $v \in V$, and for all manipulations $G^{\prime} \in \mathcal{M}(G, v): \mid\left\{x \in V^{\prime} \mid v \prec \prec_{G^{\prime}}^{F}\right.$ $x\}|\geq|\left\{x \in V \mid v \prec_{G}^{F} x\right\} \mid$; and if $\left|\left\{x \in V^{\prime} \mid v \prec_{G^{\prime}}^{F} x\right\}\right|=\left|\left\{x \in V \mid v \prec_{G}^{F} x\right\}\right|$ then $\left|\left\{x \in V^{\prime} \mid v \simeq_{G^{\prime}}^{F} x\right\}\right| \geq\left|\left\{x \in V \mid v \simeq_{G}^{F} x\right\}\right|$.

In [4] and [5], we considered manipulation by modification of an agent's outgoing links. Such outgoing link manipulation can be defined as:

$$
\mathcal{M}_{\text {out }}(V, E, v)=\left\{\left(V, E^{\prime}\right) \mid \forall u \in V \backslash\{v\}: \forall u^{\prime} \in V:\left(u, u^{\prime}\right) \in E \Leftrightarrow\left(u, u^{\prime}\right) \in E^{\prime}\right\} .
$$

The outgoing link manipulation $\mathcal{M}_{\text {out }}$ is actually a special kind of manipulation in the sense that the agent can perform the manipulation in both directions.

Definition 3.9. A manipulation $\mathcal{M}$ is called reversible if for all $G=(V, E) \in \mathbb{G}$, for all $v \in V$, and for all $G^{\prime} \in \mathcal{M}(G, v): G \in \mathcal{M}\left(G^{\prime}, v\right)$.

Reversible manipulations are important due to the following simple fact:

Fact 3.10. Let $\mathcal{M}$ be a reversible manipulation and let $F$ be a PRS . F satisfies strong incentive compatibility under $\mathcal{M}$ if and only if for all graphs $G=(V, E)$, for all sources $s \in V$, for all vertices $v \in V$, and for all manipulations $G^{\prime} \in \mathcal{M}(G, v): \mid\{x \in$ $\left.V^{\prime} \mid v \prec{ }_{G^{\prime}}^{F} x\right\}|=|\left\{x \in V \mid v \prec_{G}^{F} x\right\} \mid$ and $\left|\left\{x \in V^{\prime} \mid v \simeq_{G^{\prime}}^{F} x\right\}\right|=\left|\left\{x \in V \mid v \simeq_{G}^{F} x\right\}\right|$.

\footnotetext{
${ }^{2}$ In [4], we have defined the notion of a utility function $u_{n}: \mathbb{N} \mapsto \mathbb{R}$ that for every graph size $n$ maps the number of agents ranked below a specific agent in a strict ranking to a utility value, and we assumed such utility functions are nondecreasing. If we further assume that $u_{n}(i)=u_{m}(i+n-m)$ for all $0<i<m<n$, that is, an agent's utility in a strict ranking depends only on the number of agents ranked above it, we can show that our current defintion of stong incentive compatibility is equivalent to the one in [4].
} 
Therefore, in a PRS that is incentive compatible under a reversible manipulation an agent cannot change its rank at all by performing a manipulation.

Another type of manipulation, considered by [11] is concerned with the generation of fraudulent identities in order to manipulate one's rank. Their setting considered weighted edges, as opposed to our setting where the edges are binary. However, we can adapt their sybil form of manipulation by simply removing these weghts.

A sybil manipulation, or sybling strategy is a manipulation in which an agent controlling one vertex $v$ in the graph can create any number of fraudulent identities (or sybils) and freely manipulate the links among these sybils, while maintaining the same set of incoming and outgoing links (possibly duplicated) among the sybil group as a whole.

Thus, we can define the sybil manipulation as:

$$
\begin{aligned}
\mathcal{M}_{\text {sybil }}(V, E, v)= & \left\{\left(V^{\prime}, E^{\prime}\right) \mid\right. \\
& V \subseteq V^{\prime} \wedge \forall u, u^{\prime} \in V \backslash\{v\}:\left(u, u^{\prime}\right) \in E \Leftrightarrow\left(u, u^{\prime}\right) \in E^{\prime} \wedge \\
& P_{G}(v) \backslash\{v\}=(V \backslash\{v\}) \cap \bigcup_{u \in V^{\prime} \backslash V \cup\{v\}} P_{G^{\prime}}(u) \wedge \\
& \left.S_{G}(v) \backslash\{v\}=(V \backslash\{v\}) \cap \bigcup_{u \in V^{\prime} \backslash V \cup\{v\}} S_{G^{\prime}}(u)\right\} .
\end{aligned}
$$

We can also consider the combined manipulation of the two, which is not the same as the simple union of these manipulations:

$$
\begin{aligned}
\mathcal{M}_{\text {both }}(V, E, v)= & \left\{\left(V^{\prime}, E^{\prime}\right) \mid\right. \\
& V \subseteq V^{\prime} \wedge \forall u, u^{\prime} \in V \backslash\{v\}:\left(u, u^{\prime}\right) \in E \Leftrightarrow\left(u, u^{\prime}\right) \in E^{\prime} \wedge \\
& P_{G}(v) \backslash\{v\}=(V \backslash\{v\}) \cap \bigcup_{u \in V^{\prime} \backslash V \cup\{v\}} P_{G^{\prime}}(u) .
\end{aligned}
$$

It turns out that strong incentive compatibility under both outgoing edge and sybling manipulations is equivalent to strong incentive compatibility under the combined manipulation:

Fact 3.11. Let $F$ be a PRS. F satisfies strong incentive compatibility under $\mathcal{M}_{\text {out }}$ and under $\mathcal{M}_{\text {sybil }}$ if and only if it satisfies strong incentive compatibility under $\mathcal{M}_{\text {both }}$.

Proof. The "if" direction is trivial. For the "only if" direction, let $G=(V, E)$ be a graph and $v \in V$. Consider a manipulation $\left(V^{\prime}, E^{\prime}\right) \in \mathcal{M}_{\text {both }}(V, E, v)$. Let $U=\left\{x \mid \exists u \in V^{\prime} \backslash V \cup\{v\}:(u, x) \in E^{\prime}\right\}$. Let $E^{\prime \prime}=E \backslash\{(v, x) \mid x \in V\} \cup\{(v, x) \mid x \in$ $U\}$. Now $\left(V, E^{\prime \prime}\right) \in \mathcal{M}_{\text {out }}(V, E, v)$ and $\left(V^{\prime}, E^{\prime}\right) \in \mathcal{M}_{\text {sybil }}\left(V, E^{\prime \prime}, v\right)$, and due to strong incentive compatibility under these manipulations, $F$ also satisfies strong incentive compatibility under manipulation $\left(V^{\prime}, E^{\prime}\right)$ and indeed under any manipulation in $\mathcal{M}_{\text {both }}$.

\subsection{Satisfication}

We will now demonstrate the aforementioned axioms by showing which axioms are satisfied by the PRSs mentioned in Section 2.2. 
Proposition 3.12. The distance PRS $F_{D}$ satisfies self confidence, ranked IIA, transitivity, and strong incentive compatibility under $\mathcal{M}_{\text {both }}$, but does not satisfy strong transitivity.

Proof. Self-confidence is satisfied by definition of $F_{D} . F_{D}$ satisfies RIIA, because it ranks every comparison profile in the connected section consistently according to the following rule:

$$
\left(a_{1}, a_{2}, \ldots, a_{n}\right) \preccurlyeq\left(b_{1}, b_{2}, \ldots, b_{m}\right) \Leftrightarrow a_{n} \leq b_{m} .
$$

That is, any two vertices are compared according to their strongest predecessor. $F_{D}$ satisfies strong quasi transitivity, because the ranking of the profiles above is consistent with strong quasi transitivity. The unconnected vertices are all equal to each other and weaker than the connected vertices which is also true for their predecessors, and thus strong quasi transitivity is satisfied.

To prove that $F_{D}$ satisfies strong incentive compatibility, note the fact that an agent $x$ cannot modify the shortest path from $s$ to $x$ by changing its outgoing links or adding sybils since any such shortest path necessarily does not include $x$ or its sybils (except as target). Moreover, $x$ or its sybils cannot change the shortest path to any agent $y$ with $d(s, y) \leq d(s, x)$, because $x$ and its sybils are necessarily not on the shortest path from $s$ to $y$. Therefore, the amount of agents ranked above $\mathrm{x}$ and its sybils and the amount of agents ranked equal to $x$ or its sybils cannot decrease due to $x$ 's manipulations.

To prove $F_{D}$ does not satisfy strong transitivity, consider the graph in Figure 1a. In this graph, $x$ and $y$ are ranked the same, even though $P(x) \subsetneq P(y)$, in contradiction to strong transitivity.

Proposition 3.13. The Personalized PageRank ranking systems satisfy self confidence if and only if the damping factor is set to more than $\frac{1}{2}^{3}$. Moreover, Personalized PageRank does not satisfy weak transitivity, ranked IIA or strong incentive compatibility under $\mathcal{M}_{\text {out }}$ or $\mathcal{M}_{\text {sybil }}$ for any damping factor.

Proof. To prove the that PPR does not satisfy self-confidence for $d \leq \frac{1}{2}$, consider the graph in Figure 1b. For any damping factor $d$, the PPR will be $P P R(s)=d$ and $P P R(x)=1-d$. If $d \leq \frac{1}{2}$ then $P P R(s) \leq P P R(x)$ and thus $s \preceq^{P P R_{d}} x$, in contradiction to the self confidence axiom.

PPR satisfies self-confidence for $d>\frac{1}{2}$ because then $\operatorname{PPR}(s) \geq d>\frac{1}{2}$, while for all $v \in V \backslash\{s\}, \operatorname{PPR}(v) \leq 1-d<\frac{1}{2}$.

To prove that PPR does not satisfy strong quasi transitivity and ranked IIA, consider the graph in Figure 1c. The PPR of this graph for any damping factor $d$ is as follows: $\operatorname{PPR}(s)=d ; \quad \operatorname{PPR}(a)=\frac{d(1-d)}{2} ; \operatorname{PPR}(b)=\frac{d(1-d)^{2}}{4} ; \operatorname{PPR}(c)=\frac{d(1-d)^{2}}{2}$. Therefore, the ranking of this graph is: $b \prec c \prec a \prec s$. Quasi transitivity is violated because $b \prec c$ even though $P(b)=P(c)=a$. This also violates ranked IIA because the ranking profile $\langle(1),(1)\rangle$ must be ranked as equal due to trivial comparisons such as $a$ and $a$.

\footnotetext{
${ }^{3}$ If we do not allow self-loops this bound becomes $(\sqrt{5}-1) / 2 \approx 0.618$.
} 


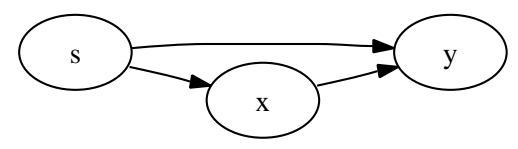

(a)

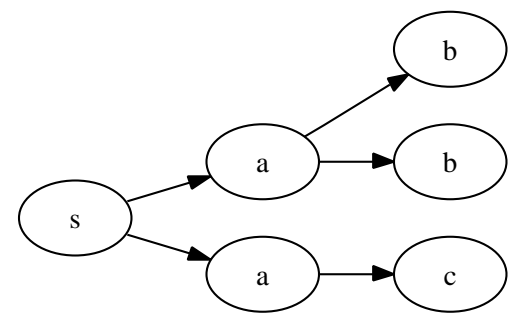

(b)

(c)
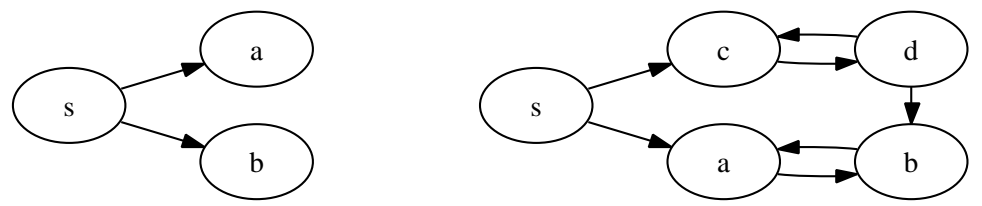

(d)

(e)

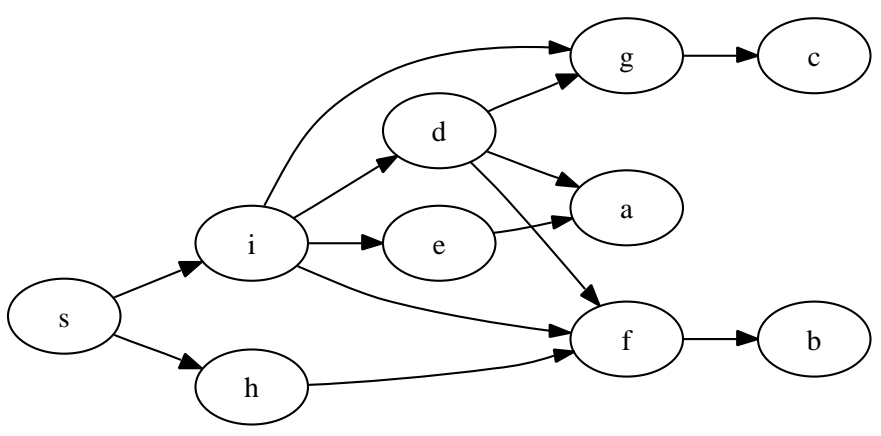

(f)

Figure 1: Graphs proving PRS do not satisfy axioms. 
Strong incentive compatibility under $\mathcal{M}_{\text {out }}$ is not satisfied, because in the graph in Figure 1c, if any of the $b$ agents $b^{\prime}$ would have voted for themselves, they would have been ranked $b \prec b^{\prime} \prec c \prec a \prec s$, which is a strict increase in $b^{\prime}$ rank.

To show that strong incentive compatibility under $\mathcal{M}_{\text {sybil }}$ is not satisfied, consider the graph in Figure 1d. Note that $a \simeq b \prec s$ in this graph. Consider the manipulation by $a$ where a sybil $a^{\prime}$ is added along with the edges $\left\{\left(s, a^{\prime}\right),\left(a^{\prime}, a\right)\right\}$. In this case, the PageRank value of $b$ would be $\frac{1}{3}(1-d) d$ while the PageRank value of $a$ will be $\frac{(1-d)+1}{3}(1-d) d$. Therefore, $b \prec a \prec s$ in the manipulated graph, and thus strong incentive compatibility is not satisfied.

It is interesting to note that although Personalized Pagerank does not satisfy strong incentive compatibility under $\mathcal{M}_{\text {sybil }}$, a weighted version of Personalized PageRank is in fact sybilproof with regard to the weighted definition of sybilproofness presented in [11].

Strong transitivity is also satisfied by a natural PRS — the $\alpha$-Rank system:

Proposition 3.14. The $\alpha$-Rank system satisfies self confidence and strong transitivity, but does not satisfy ranked IIA or strong incentive compatibility under $\mathcal{M}_{\text {out }}$ or $\mathcal{M}_{\text {sybil. }}$.

Proof. To show $\alpha$-Rank satisfies self confidence, note that by definition $r_{G, s}(s) \geq 1$. Assume for contradiction that $\max _{v \neq s} r_{G, s}(v) \geq 1$. Then,

$$
\begin{aligned}
r_{G, i}(s) & \leq 1+\alpha \sum_{v \in V} r_{G, s}(v) \\
& \leq 1+\alpha\left[(n-1) \max _{v \neq s} r_{G, s}(v)+r_{G, i}(s)\right] \\
r_{G, i}(s) & \leq \frac{1}{1-\alpha}+\frac{\alpha}{1-\alpha}(n-1) \max _{v \neq s} r_{G, s}(v) \leq \\
& \leq 2+\max _{v \neq s} r_{G, s}(v) \\
\max _{v \neq s} r_{G, s}(v) & \leq \alpha^{n}+\alpha \sum_{v \in V} r_{G, s}(v) \\
{\left[1-\frac{n^{n}}{n^{2}}\right] \max _{v \neq s} r_{G, s}(v) } & \leq \frac{2}{n^{2}}+\frac{1}{n^{2 n}} \\
n^{2}-n & \leq 2+1 / n^{2 n-2} \\
n^{2}-n-1 / n^{2 n-2} & \leq 2 \\
2 \leq n(n-1) & <2
\end{aligned}
$$

To prove $\alpha$-Rank satisfies strong transitivity, consider two vertices $a, b \in V \backslash\{s\}$ and a function $f: P(a) \mapsto P(b)$ such that $v \preceq f(v)$ for all $v \in P(a)$. Then,

$$
r_{G, s}(a) / \alpha-\alpha^{n}=\sum_{v \in P(a)} r_{G, s}(v) \leq \sum_{v \in f(P(a))} r_{G, s}(v) \leq
$$




$$
\leq \sum_{v \in P(b)} r_{G, s}(v)=r_{G, s}(b) / \alpha-\alpha^{n},
$$

which implies $a \preceq b$. If for some $v \in P(a): v \prec f(v)$, or if $f$ is not onto, then the first or the second inequality respectively in (1) above is strict, which implies $a \prec b$, as required.

To prove $\alpha$-Rank does not satisfy strong incentive compatibility under $\mathcal{M}_{\text {out }}$, consider the graph in Figure 1e. In this graph $\alpha$-Rank ranks $d \prec b$. However, if $d$ removes the link to $b$ they will be ranked equally and thus reducing the number of agents stronger than $d$. To prove $\alpha$-Rank does not satisfy strong incentive compatibility under $\mathcal{M}_{\text {sybil }}$, consider again the graph in Figure 1e. Agent $c$ is ranked below agent $b$ in this graph. However, she can duplicate herself and add edges $\left(c, c^{\prime}\right)$ and $\left(c^{\prime}, c\right)$ to be ranked above $b$ thus decreasing the number of agents ranked better than herself.

To prove $\alpha$-Rank does not satisfy RIIA, consider the graph in Figure 1f. It is easy to calculate the following $\alpha$-Rank values:

$$
\begin{aligned}
r(s) & =1 \\
r(i)=r(h) & =\alpha+\alpha^{10} \\
r(d)=r(e) & =\alpha^{2}+\alpha^{10}+\alpha^{11} \\
r(f) & =2 \alpha^{2}+\alpha^{3}+\alpha^{10}+3 \alpha^{11}+\alpha^{12} \\
r(g) & =\alpha^{2}+\alpha^{3}+\alpha^{10}+2 \alpha^{11}+\alpha^{12} \\
r(a) & =2 \alpha^{3}+\alpha^{10}+2 \alpha^{11}+2 \alpha^{12} \\
r(b) & =2 \alpha^{3}+\alpha^{4}+\alpha^{10}+\alpha^{11}+3 \alpha^{12}+\alpha^{13} \\
r(c) & =\alpha^{3}+\alpha^{4}+\alpha^{10}+\alpha^{11}+2 \alpha^{12}+\alpha^{13} .
\end{aligned}
$$

Therefore, this graph is ranked $c \prec a \prec b \prec d \simeq e \prec g \prec f \prec i \simeq h \prec s$. Note that $(a, b)$ and $(a, c)$ both satisfy the profile $\langle(1,1),(2)\rangle$, however $a \prec b$ and $c \prec a$ in contradiction to RIIA.

\section{A Characterization Theorem}

Our main result is a full characterization of the PRSs that satisfy the axioms above. We will see that these systems are the generalized strong count systems. Strong count rank agents based on their strongest predecessors, breaking ties according to the number of equal strongest predecessors the agents have. The function $r$ below determines how such ties are broken. As $s$ is stronger than all other agents, the strongest predecessor of each agent in $V_{s} \backslash\{s\}$ must be closer to $s$.

The strong count system is formally defined as follows:

Definition 4.1. Let $r: \mathbb{N} \mapsto \mathbb{N}$ be a monotone nondecreasing function such that $r(i) \leq i$ for all $i \in \mathbb{N}$. The strong count system $S C_{r}$ is recursively defined as follows: First of all, $y \simeq y^{\prime} \prec x \prec s$ for all $x \in V_{s} \backslash\{s\}$ and $y, y^{\prime} \in V \backslash V_{s}$. For $x \in V_{s} \backslash\{s\}$, denote $P^{\prime}(x)=P(x) \cap\{y \mid d(s, y)<d(s, x)\}$, and $P_{\max }(x)=\left\{y \mid y \in P^{\prime}(x), \forall z \in\right.$ 
$\left.P^{\prime}(x): z \preceq{ }^{S C_{r}} y\right\}$. Now for $a, b \in V_{s} \backslash\{s\}$ :

$$
\begin{aligned}
a \preceq^{S C_{r}} b \Leftrightarrow & \left(\exists x \in P_{\max }(a), y \in P_{\max }(b): x \prec^{S C_{r}} y\right) \vee \\
\vee[\quad & \left(\forall x \in P_{\max }(a), y \in P_{\max }(b): x \simeq^{S C_{r}} y\right) \wedge \\
& \wedge\left(\left(r\left(\left|P_{\max }(a)\right|\right) \leq r\left(\left|P_{\max }(b)\right|\right)\right)\right] .
\end{aligned}
$$

The strong count systems rank based on the strongest predecessor's rank and then break ties based on the number of strongest predecessors. Unconnected vertices are equally ranked at the bottom. Note that for $r \equiv 1$, the Strong Count PRS is exactly the distance system.

Our main result claims that these strong count systems are the only systems that satisfy all aforementioned axioms.

Theorem 4.2. Let $F$ be a PRS. The following three statements are equivalent:

1. F is a strong count system for some $r$.

2. F satisfies self confidence, strong quasi transitivity, ranked IIA and strong incentive compatibility under $\mathcal{M}_{\text {out }}$.

3. F satisfies self confidence, strong quasi transitivity, ranked IIA and strong incentive compatibility under $\mathcal{M}_{\text {both }}$.

We begin our proof by showing that the strong count systems do in fact satisfy all these axioms.

Proof. ( $(\Rightarrow 3)$ : Let $r$ be a monotone nondecreasing function such that $r(x) \leq x . S C_{r}$ satisfies self confidence by definition.

To show that $S C_{r}$ satisfies RIIA and strong quasi transitivity on elements of $V_{s}$, we will show that it ranks any profile $p=\left\langle\left(a_{1}, \ldots, a_{n}\right) ;\left(b_{1}, \ldots, b_{m}\right)\right\rangle$ as follows: Let $c_{a}=\max \left\{i \in \mathbb{N} \mid a_{n-i}=a_{n-i+1}=\cdots=a_{n}\right\}$ and $c_{b}=\max \left\{i \in \mathbb{N} \mid b_{m-i}=\right.$ $\left.b_{m-i+1}=\cdots=b_{m}\right\}$.

$$
\begin{aligned}
f(p)=1 & \Leftrightarrow \quad\left(a_{n}<b_{m}\right) \vee \\
& \vee \quad\left[\left(a_{n}=b_{m}\right) \wedge\left(r\left(c_{a}\right) \leq r\left(c_{b}\right)\right)\right]
\end{aligned}
$$

This almost follows from the recursive definition of $S C_{r}$, however it remains to show that $\forall x, y \in V: d(s, x)<d(s, y) \Rightarrow x \prec^{S C} y$. This can be proven by induction on $d(s, y)$. If $y=s$ this is trivial by definition. Otherwise, by the assumption of induction, $\exists x^{\prime} \in P_{\max }(x), y^{\prime} \in P_{\max }(y): x^{\prime} \prec^{S C} y^{\prime}$ and thus by the recursive definition, $x \prec^{S C} y$.

Strong quasi transitivity involving elements in $V \backslash V_{s}$ and elements either in $V \backslash V_{s}$ or in $V_{s} \backslash\{s\}$ is satisfied because for all $x \in V \backslash V_{s}$ and $y \in V \backslash\{s\}$ we have $x \preceq y$ (by definition) and if $x \prec y$ then $y \in V_{s} \backslash\{s\}$ and thus there is some $y^{\prime} \in P(y)$ such that for all $x^{\prime} \in P(x): x^{\prime} \preceq y^{\prime}$.

With regard to the strong incentive compatibility under $\mathcal{M}_{\text {both }}$, due to the distance feature proven above, all sybils of $v$ will be strictly weaker than the vertices with smaller distance from $s$. Furthermore, any other vertices that were stronger than $v$ in 
the original graph will be stronger than any of $v$ 's sybils, due to the fact that the relative rank of two vertices is determined only based on incoming links from vertices closer to $s$, and more incoming edges cannot decrease an agent's rank. By the same logic, vertices which were equal to $v$ in the original graph, will either be stronger or equal to $v$ in the manipulated graph.

In order to prove the hard direction of Theorem $4.2(2 \Rightarrow 1)$, we will first show that a strong notion of transitivity is implied by the axioms:

Definition 4.3. Let $F$ be a PRS. We say that $F$ satisfies weak maximum transitivity if for all graphs $G=(V, E)$, for all sources $s \in V$ and for all vertices $v_{1}, v_{2} \in V_{s}$ : Let $m_{1}, m_{2}$ be the maximally ranked vertices in $P\left(v_{1}\right), P\left(v_{2}\right)$ respectively. Assume $m_{1} \prec m_{2}$. Then, $v_{1} \prec v_{2}$.

Lemma 4.4. Let $F$ be a PRS that satisfies self confidence, strong quasi transitivity, RIIA and strong incentive compatibility. Then, F satisfies weak maximum transitivity.

Proof. In order to show that $F$ satisfies weak maximum transitivity, we will show that for every comparison profile the ranking must be consistent with weak maximum transitivity. Let $p=\left\langle\left(a_{1}, a_{2}, \ldots, a_{k}\right),\left(b_{1}, b_{2}, \ldots, b_{l}\right)\right\rangle$ be a comparison profile where $a_{k} \neq b_{l}$. Assume wlog that $b_{l}<a_{k}$ and assume for contradiction that $\left\langle\left(a_{1}, a_{2}, \ldots, a_{k}\right) \preceq\left(b_{1}, b_{2}, \ldots, b_{l}\right)\right\rangle$. Consider the graph $G=(V, E)$ defined as follows:

$$
\begin{aligned}
V= & \{s, a, b\} \cup\left\{u_{i}^{j} \mid i \in\{1, \ldots, \max (k, l)\} ; j \in\left\{0, \ldots, a_{k}\right\}\right\} \\
E= & \left\{\left(u_{i}^{j}, u_{i}^{j-1}\right) \mid i \in\{1, \ldots, \max (k, l)\} ; j \in\left\{1, \ldots, a_{k}\right\}\right\} \cup \\
& \cup\left\{\left(s, u_{i}^{b_{l}}\right) \mid i \in\{1, \ldots, \max (k, l)\}\right\} \cup \\
& \cup\left\{\left(u_{i}^{j}, a\right) \mid a_{i}=j\right\} \cup\left\{\left(u_{i}^{j}, b\right) \mid b_{i}=j\right\} .
\end{aligned}
$$

Figure 2 contains such a graph for the profile $\langle(1,4),(2,2,3)\rangle$.

Note that by strong quasi transitivity and self confidence, for all $i, i^{\prime}, j, j^{\prime}: u_{i}^{j} \preceq u_{i^{\prime}}^{j^{\prime}}$ iff, $j \leq j^{\prime}$. Therefore, we will use $u^{j}$ to denote any $u_{i}^{j}$. By the construction of $G, a$ and $b$ satisfy $p$. Thus, from our assumption, $a \preceq b$.

By strong quasi transitivity, $a \succeq u^{b_{l}}$, and thus from our assumption also $b \succeq u^{b_{l}}$. Now consider the point of view of agent $u_{l}^{b_{l}}$. She can perform a manipulation by not voting for $b$. This manipulation must not change her relative rank, as it is in $\mathcal{M}_{\text {out }}$. As the relative ranks of the $u_{i}^{j}$ agents and $s$ are unaffected by this manipulation, it cannot affect the ranks of $a$ and $b$ relative to $u_{l}^{b_{l}}$, and thus after the edge $\left(u_{l}^{b_{l}}, b\right)$ is removed, we still have $b \succeq u_{l}^{b_{l}}$. We can repeat this process for all $i=b_{l}, \ldots, 2$, with the result that in the graph $G^{\prime}$ for the profile $\left\langle\left(a_{1}, a_{2}, \ldots, a_{k}\right),\left(b_{1}\right)\right\rangle, b \succeq u^{b_{2}} \succeq u^{b_{1}}$. However, by strong quasi transitivity, $b \simeq_{G^{\prime}} u^{b_{1}-1} \prec_{G^{\prime}} u^{b_{1}} \preceq_{G^{\prime}} b$, which is a contradiction.

We can now prove the hard direction of Theorem 4.2. 


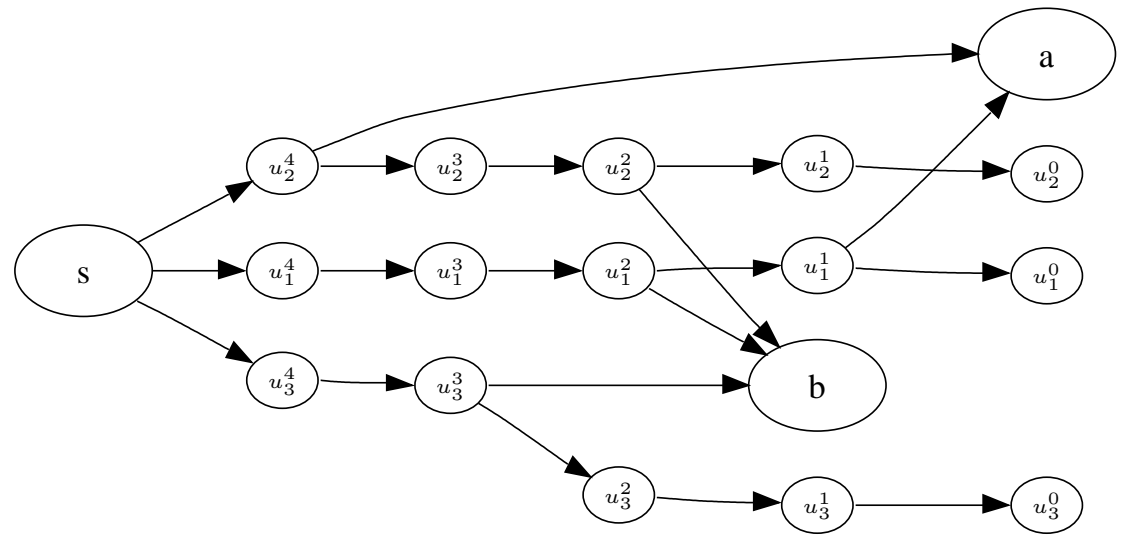

Figure 2: Example of graph from proof of Lemma 4.4.

Proof. (Theorem 4.2: $2 \Rightarrow 1$ ) Given Lemma 4.4, it remains to look at profiles $\left\langle\left(a_{1}, a_{2}, \ldots, a_{k}\right),\left(b_{1}, b_{2}, \ldots, b_{l}\right)\right\rangle$ where $a_{k}=b_{l}$. Denote $M=a_{k}=b_{l}$. Let $p$ be such a profile. Denote $x_{a}=\mid\left\{n \mid a_{n}=\right.$ $M\} \mid$ and similarly $x_{b}=\left|\left\{n \mid b_{n}=M\right\}\right|$. These values denote the number of strongest predecessors $a$ and $b$ have in profile $p$.

We will now prove by induction on $k+l-x_{a}-x_{b}$ that $F$ ranks $p$ the same as it ranks $\left\langle(\underbrace{1, \ldots, 1}_{x_{a} \text { times }}),(\underbrace{1, \ldots, 1}_{x_{b} \text { times }})\right\rangle$. If $k+l-x_{a}-x_{b}=0$, then $a_{1}=a_{k}=b_{1}=b_{l}$, and thus the requirement is trivially satisfied. Otherwise, we assume correctness for $k+l-x_{a}-x_{b}-1$. Further assume wlog that $a_{1} \neq a_{k}$. Denote $r=a_{k-x_{a}}$ and $y_{a}=\left|\left\{n \mid a_{n}=r\right\}\right|$.

We shall now consider two cases:

- If $b_{1}=b_{l}$ or $a_{k-x_{a}} \neq b_{l-x_{b}}$. If $b_{1} \neq b_{l}$, then further assume wlog that $a_{k-x_{a}}>$ $b_{l-x_{b}}$. Consider the graph $G=(V, E)$ defined as follows:

$$
\begin{aligned}
V= & \{s, a\} \cup\left\{b^{1}, \ldots, b^{y_{a}}\right\} \cup \\
& \cup\left\{u_{i}^{j} \mid i \in\{1, \ldots, \max (k, l)\} ; j \in\{0, \ldots, M\}\right\} \\
E= & \left\{\left(u_{i}^{j}, u_{i}^{j-1}\right) \mid i \in\{1, \ldots, \max (k, l)\} ; j \in\{1, \ldots, M\}\right\} \cup \\
& \cup\left\{\left(s, u_{i}^{M}\right) \mid i \in\{1, \ldots, \max (k, l)\}\right\} \cup\left\{\left(u_{i}^{j}, a\right) \mid a_{i}=j \neq r\right\} \cup \\
& \cup\left\{\left(u_{i}^{j}, b^{n}\right) \mid b_{i}=j, n=1, \ldots, y_{a}\right\} \cup\left\{\left(b^{n}, a\right) \mid n=1, \ldots, y_{a}\right\} .
\end{aligned}
$$

Figure 3 contains such a graph for the profile $\langle(1,3,3,4),(1,2,4,4)\rangle$. Note that by strong quasi transitivity and self confidence, for all $i, i^{\prime}, j, j^{\prime}: u_{i}^{j} \preceq u_{i^{\prime}}^{j^{\prime}}$ iff ,$j \leq j^{\prime}$. Therefore, we will use $u^{j}$ to denote any $u_{i}^{j}$. Similarly, all $b^{n}$ are equal to each other, and by weak maximum transitivity (Lemma 4.4), $u^{M-1} \preceq a, b \prec u^{M}$ (we will similarly use $b$ to denote any $b^{n}$ ). Therefore, $a$ and $b$ satisfy $p$. Now consider the following manipulation by $b^{1}$ : Removing the outgoing edge to $a$. This manipulation is in $\mathcal{M}_{\text {out }}$ and thus should not change the relative rank of $b^{1}$. 


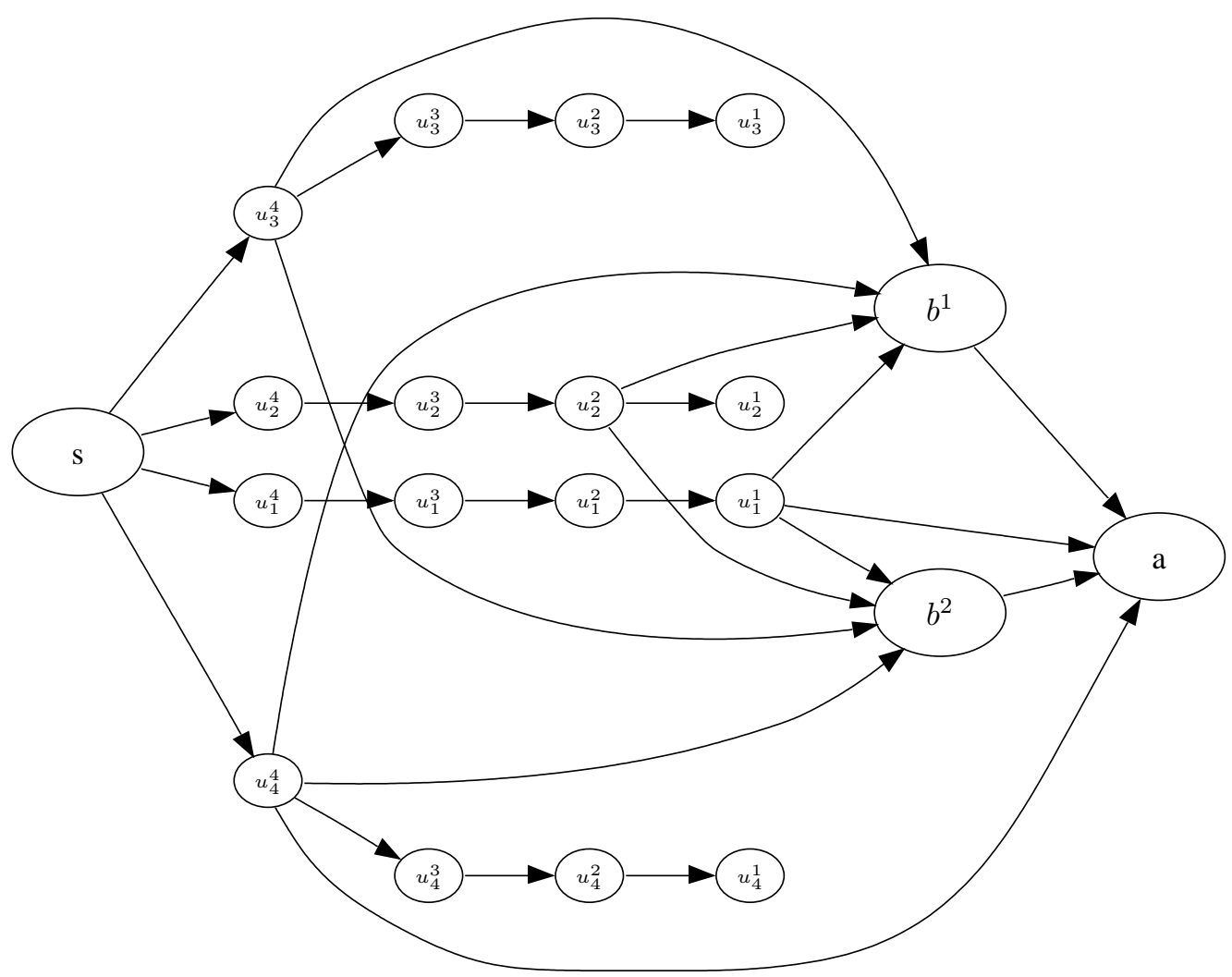

Figure 3: Example graph from the proof of Theorem 4.2 case 1. 
Note that $b^{1}$ 's predecessors remain the same and equal to the ones of $b^{2}, \ldots, b^{y_{a}}$, and all $b^{n}$ remain equal. We must now show that for every allowable relative ranking of $u^{M-1}, a$, and $b$ the manipulation cannot change $a$ and $b$ 's relative rank. We will do this by considering all cases:

\begin{tabular}{|c|c|c|}
\hline Ordering & \# Vertices equal to $b$ & \# Vertices stronger than $b$ \\
\hline \hline$u^{M-1} \simeq b \prec a$ & $y_{a}+\max (k, l)$ & $(M-r) \cdot \max (k, l)+2$ \\
\hline$u^{M-1} \prec b \prec a$ & $y_{a}$ & $(M-r) \cdot \max (k, l)+2$ \\
\hline$u^{M-1} \simeq a \simeq b$ & $y_{a}+\max (k, l)+1$ & $(M-r) \cdot \max (k, l)+1$ \\
\hline$u^{M-1} \prec a \simeq b$ & $y_{a}+1$ & $(M-r) \cdot \max (k, l)+1$ \\
\hline$u^{M-1} \simeq a \prec b$ & $y_{a}$ & $(M-r) \cdot \max (k, l)+1$ \\
\hline$u^{M-1} \prec a \prec b$ & $y_{a}$ & $(M-r) \cdot \max (k, l)+1$ \\
\hline
\end{tabular}

We see that any change in the relation between $a$ and $b$ will surely change $b$ 's rank in a way that is not strategyproof.

We have shown that profile $p$ must be ranked the same as the profile

$$
\left\langle\left(a_{1}, a_{2}, \ldots, a_{k-x_{a}-1}, a_{k-x_{a}+1}, \ldots, a_{k}\right),\left(b_{1}, b_{2}, \ldots, b_{l}\right)\right\rangle \text {, }
$$

which by the assumption of induction gives us the desired result.

- Otherwise, $a_{k-x_{a}}=b_{l-x_{b}}$. Denote $y_{b}=\left|\left\{n \mid b_{n}=r\right\}\right|$ and assume wlog that $y_{b} \geq y_{a}$. Consider the graph $G=(V, E)$ defined as follows:

$$
\begin{aligned}
V= & \{s, a\} \cup\left\{b^{0}, \ldots, b^{y_{b}}\right\} \cup \\
& \cup\left\{u_{i}^{j} \mid i \in\{1, \ldots, \max (k, l)\} ; j \in\{0, \ldots, M\}\right\} \\
E= & \left\{\left(u_{i}^{j}, u_{i}^{j-1}\right) \mid i \in\{1, \ldots, \max (k, l)\} ; j \in\{1, \ldots, M\}\right\} \cup \\
& \cup\left\{\left(s, u_{i}^{M}\right) \mid i \in\{1, \ldots, \max (k, l)\}\right\} \cup\left\{\left(u_{i}^{j}, a\right) \mid a_{i}=j \neq r\right\} \cup \\
& \cup\left\{\left(u_{i}^{j}, b^{n}\right) \mid b_{i}=j \neq r, n=0, \ldots, y\right\} \cup \\
& \cup\left\{\left(b^{n}, a\right) \mid n=1, \ldots, y_{a}\right\} \cup\left\{\left(b^{n}, b^{m}\right) \mid n \neq m \in\left\{0, \ldots, y_{b}\right\}\right\} .
\end{aligned}
$$

Figure 4 contains such a graph for the profile $\langle(1,1,2,2),(1,1,1,2)\rangle$. As before, for all $i, i^{\prime}, j, j^{\prime}: u_{i}^{j} \preceq u_{i^{\prime}}^{j^{\prime}}$ iff,$j \leq j^{\prime}$ and we will use $u^{j}$ to denote any $u_{i}^{j}$. All $b^{n}$ are equal to each other because if wlog $b^{1} \prec b^{2}$ then $b^{1}$ 's predecessors will be stronger than $b^{2}$ 's predecessors and thus by strong quasi transitivity $b^{2} \preceq$ $b^{1}$. Again, by weak maximum transitivity, $u^{M-1} \preceq a, b \prec u^{M}$ and we will use $b$ to denote any $b^{n}$. Therefore, $a$ and $b$ satisfy $p$. We can again consider a manipulation by $b^{1}$ removing an edge to $a$, again all $b^{n}$ remain equal and as before the manipulation cannot change $a$ and $b$ 's relative rank, and when again applying the assumption of induction we get the desired result.

By strong quasi transitivity, profiles where all predecessors are equal are ranked $\langle 1\rangle \preceq$ $\langle 1,1\rangle \preceq \cdots$. When considering the result above, we conclude any two vertices should be weakly ranked according to the number of strongest predecessors they have, and by RIIA the tie-breaking rule must be universal. 


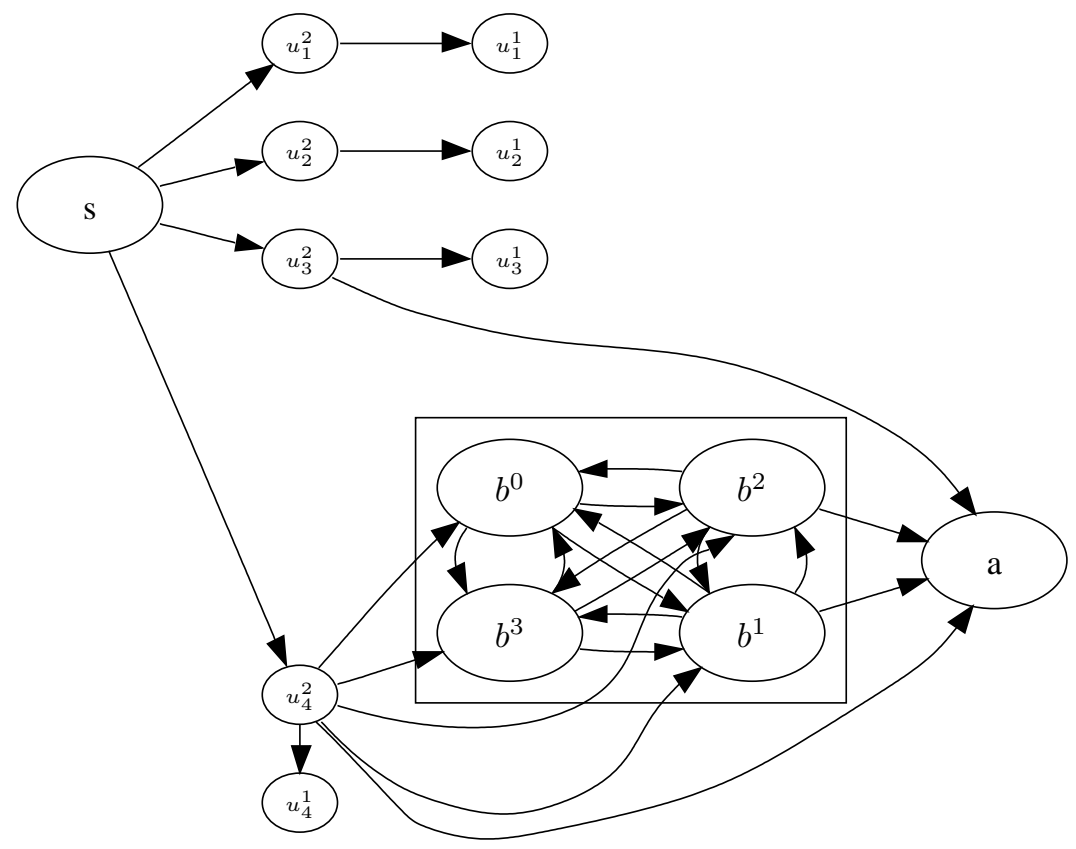

Figure 4: Example graph from the proof of Theorem 4.2 case 2.

It remains to show that vertices in $V \backslash V_{s}$ will be ranked equally and strictly weaker than those in $V_{s}$. Let $m \in V_{s}$ be a minimally ranked vertex in $V_{s}$. Consider a manipulation by $m$ adding edges to all vertices in $V \backslash V_{s}$. By the above proof, all vertices in $V \backslash V_{s}$ will be equally ranked weaker than $m$. As $m$ does not worsen its position by performing this manipulation and the internal ranking in $V_{s}$ does not change we conclude that in any graph all vertices in $V \backslash V_{s}$ must be ranked strictly weaker than those in $V_{s}$.

We can show the vertices in $V \backslash V_{s}$ are ranked equally by induction on the number of edges between them. If there are no such edges, then by strong quasi transitivity, the requirement is satisfied. Otherwise, consider an edge $\left(v_{1}, v_{2}\right)$ such that $v_{1}, v_{2} \in V \backslash V_{s}$. A manipulation by $v_{1}$ adding this edge must retain its position and thus all agents in $V \backslash V_{s}$ must be ranked equally.

We have shown that all vertices must be ranked according to strong count and thus the system must be a strong count system.

\section{Relaxing the Axioms}

We shall now prove the conditions in Lemma 4.4 (and thus also in Theorem 4.2(2)) are all necessary by showing PRSs that satisfy each three of the four conditions, but do not satisfy weak maximum transitivity. Some of these systems are quite artificial, while others are interesting and useful. 
Proposition 5.1. There exists a PRS that satisfies strong quasi transitivity, RIIA and strong incentive compatibility, but not self confidence nor weak maximum transitivity.

Proof. Let $F_{D}^{-}$be the PRS that ranks strictly the opposite of the depth system $F_{D}$.

That is, $v_{1} \preceq_{G, s}^{F_{D}^{-}} v_{2} \Leftrightarrow v_{2} \preceq_{G, s}^{F_{D}} v_{1}$. The proof $F_{D}^{-}$satisfies strong quasi transitivity, RIIA and strong incentive compatibility follows the proof of Proposition 3.12, with the following rule for ranking comparison profiles:

$$
\left(a_{1}, a_{2}, \ldots, a_{n}\right) \preccurlyeq\left(b_{1}, b_{2}, \ldots, b_{m}\right) \Leftrightarrow a_{1} \leq b_{1} .
$$

$F_{D}^{-}$does not satisfy self confidence, because, by definition $s$ is weaker than all other agents, and does not satisfy weak maximum transitivity because in graph from Figure 1a, $F_{D}^{-}$ranks $x$ and $y$ equally even though the strongest predecessor of $y$, which is $x$, is stronger than the strongest predecessor of $x$, which is $s$.

This PRS is highly unintuitive, as the most trusted agents are the ones furthest from the source, which is by itself the least trusted.

Relaxing strong quasi transitivity leads to a PRS that is almost trivial:

Proposition 5.2. There exists a PRS that satisfies self confidence, ranked IIA and strong incentive compatibility, but not strong quasi transitivity nor weak maximum transitivity.

Proof. Let $F$ be the $P R S$ which ranks for every $G=(V, E)$, for every source $s \in V$, and for every $v_{1}, v_{2} \in V \backslash\{s\}: v_{1} \simeq v_{2} \prec s$. That is, $F$ ranks $s$ on the top, and all of the other agents equally. $F$ trivially satisfies self confidence, RIIA and strong incentive compatibility, as $s$ is indeed stronger than all other agents and every comparison profile is ranked equally. $F$ does not satisfy strong quasi transitivity or weak maximum transitivity, because in a chain of vertices starting from $s$ all except $s$ will be ranked equally,

\subsection{Relaxing Ranked IIA}

When Ranked IIA is relaxed, we find a new ranking system that ranks according to the distance from $s$, breaking ties according to the number of shortest paths from $s$.

Notation 5.3. Let $G=(V, E)$ be some directed graph and $v_{1}, v_{2} \in V$ be some vertices, we will use $n_{G}\left(v_{1}, v_{2}\right)$ to denote the number of directed paths of minimum length between $v_{1}$ and $v_{2}$ in $G$. We will sloppily use the notations $d(v)$ and $n(v)$ to denote $d_{G}(s, v)$ and $n_{G}(s, v)$ respectively.

Definition 5.4. The Path Count PRS $F_{P}$ is defined as follows: Given a graph $G=$ $(V, E)$ and a source $s$, for all $v_{1}, v_{2} \in V \backslash\{s\}$ :

$$
\begin{aligned}
v_{1} \preceq_{G, s}^{F_{P}} v_{2} \Leftrightarrow & d_{G}\left(s, v_{1}\right)>d_{G}\left(s, v_{2}\right) \vee \\
& \left(d_{G}\left(s, v_{1}\right)=d_{G}\left(s, v_{2}\right) \wedge\right. \\
& \left.\wedge n_{G}\left(s, v_{1}\right) \leq n_{G}\left(s, v_{2}\right)\right)
\end{aligned}
$$


Proposition 5.5. The path count PRS $F_{P}$ satisfies self confidence, strong quasi transitivity and strong incentive compatibility under $\mathcal{M}_{\text {both }}$, but not ranked IIA nor weak maximum transitivity.

Proof. Self confidence is trivial as $d(s)=0<d(v)$ for all $v \neq s$.

To prove $F_{P}$ satisfies quasi transitivity consider a graph $G=(V, E)$, a source $s \in V$ and two vertices $v_{1}, v_{2} \in V \backslash\{s\}$. Assume for contradiction that $v_{2} \prec v_{1}$ and there exists a 1-1 function $f: P\left(v_{1}\right) \mapsto P\left(v_{2}\right)$ such that $v \preceq f(v)$ for all $v \in P\left(v_{1}\right)$. By the definition of $F_{P}: d\left(v_{1}\right) \leq d\left(v_{2}\right)$, but

$$
d\left(v_{1}\right)=\min _{v \in P\left(v_{1}\right)} d(v)+1 \geq \min _{v \in f\left(P\left(v_{1}\right)\right)} d(v)+1 \geq \min _{v \in P\left(v_{2}\right)} d(v)+1=d\left(v_{2}\right),
$$

and thus $d\left(v_{1}\right)=d\left(v_{2}\right)$. Now,

$$
\begin{aligned}
n\left(v_{1}\right) & =\sum_{v \in P\left(v_{1}\right) \wedge d(v)+1=d\left(v_{1}\right)} n(v) \leq \\
& \leq \sum_{v \in f\left(P\left(v_{1}\right)\right) \wedge d\left(f^{-1}(v)\right)+1=d\left(v_{1}\right)} n(v) \leq \\
& \leq \sum_{v \in P\left(v_{2}\right) \wedge d(v)+1=d\left(v_{2}\right)} n(v)=n\left(v_{2}\right) .
\end{aligned}
$$

Therefore, $v_{1} \preceq v_{2}$ in contradiction to our assumption.

For strong quasi transitivity, assume now that $v_{2} \preceq v_{1}, P\left(v_{1}\right) \neq \emptyset$, and there exists a 1-1 function $f: P\left(v_{1}\right) \mapsto P\left(v_{2}\right)$ such that $v \prec f(v)$ for all $v \in P\left(v_{1}\right)$. As above we find that $d\left(v_{1}\right)=d\left(v_{2}\right)$. Now,

$$
\begin{aligned}
n\left(v_{1}\right) & =\sum_{v \in P\left(v_{1}\right) \wedge d(v)+1=d\left(v_{1}\right)} n(v)< \\
& \sum_{v \in f\left(P\left(v_{1}\right)\right) \wedge d\left(f^{-1}(v)\right)+1=d\left(v_{1}\right)} n(v) \leq n\left(v_{2}\right),
\end{aligned}
$$

which yields $v_{1} \prec v_{2}$ in contradiction to our assumption.

To show $F_{P}$ satisfies strong incentive compatibility under $\mathcal{M}_{b o t h}$, note that a manipulation by $v$ cannot change $d(v)$ or $d\left(v^{\prime}\right) \forall v^{\prime}: d\left(v^{\prime}\right)<d(v)$. Moreover, $v$ and its sybils cannot gain any new edges from vertices closer to $v$ or change their internal edges. For this reason, $n(v)$ cannot increase and $n\left(v^{\prime}\right)$ cannot decrease for all $v^{\prime}$ s.t. $d\left(v^{\prime}\right) \leq d(v)$. Thus, $F_{P}$ does indeed satisfy strong incentive compatibility under $\mathcal{M}_{\text {both }}$.

To show $F_{P}$ does not satisfy ranked IIA nor weak maximum transitivity, consider the graph in Figure 5. $F_{P}$ ranks this graph as follows: $a \prec b \prec y \prec z \prec x \prec s$. Consider the profile $\langle(2) ;(1,1)\rangle$. If we compare $x$ and $y$ we get $(1,1) \prec(2)$, but if we compare $a$ and $b$ we get $(2) \prec(1,1)$, in violation of ranked IIA. Furthermore, the latter comparison is in violation of weak maximum transitivity, as required. 


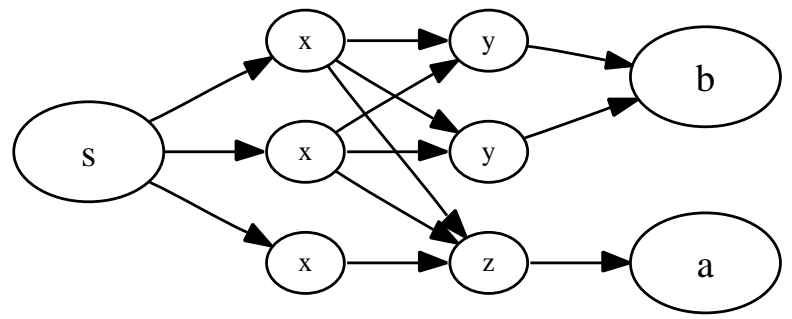

Figure 5: Proof $F_{P}$ does not satisfy axioms.

\subsection{Relaxing incentive compatibility}

When we relax incentive compatibility we find an interesting family of PRSs that rank the agents according to their in-degree, breaking ties by comparing the ranks of the strongest predecessors. These recursive in-degree systems work by assigning a rational number trust value for every vertex, that is based on the following idea: rank first based on the in-degree. If there is a tie, rank based on the strongest predecessor's trust, and so on. Loops are ranked as periodical rational numbers in base $(n+2)$ with a period the length of the loop, only if continuing on the loop is the maximally ranked option.

The recursive in-degree systems differ in the way different in-degrees are compared. Any monotone increasing mapping of the in-degrees could be used for the initial ranking. To show these systems are well-defined and that the trust values can be calculated we define these systems algorithmically as follows:

Definition 5.6. Let $r: \mathbb{N} \mapsto \mathbb{N}$ be a monotone nondecreasing function such that $r(i) \leq i$ for all $i \in \mathbb{N}$. The recursive in-degree PRS with rank function $r$ is defined as follows: Given a graph $G=(V, E)$ and source $s$,

$$
v_{1} \preceq_{G, s}^{R I D_{r}} v_{2} \Leftrightarrow \operatorname{value}_{r, s}\left(v_{1}\right) \leq \operatorname{value}_{r, s}\left(v_{2}\right),
$$

where value is defined as:

$$
\operatorname{value}_{r, s}(v)=\max _{\mathbf{a} \in \operatorname{Path}_{s}(v)} \operatorname{vp}_{r, s}(\mathbf{a})
$$

where the maximum is over the set of almost-simple paths to $v$ not passing through $s$ (but which may start at $s$ ):

$$
\begin{aligned}
\operatorname{Path}_{s}(v)=\{ & \left(v=a_{1}, a_{2}, \ldots, a_{m}\right) \mid \\
& \left(a_{m}, \ldots, a_{1}\right) \text { is a path in } G \wedge\left(a_{m-1}, \ldots, a_{1}\right) \text { is simple } \wedge \\
& \left.\forall i \in\{1 \ldots m-1\}: a_{i} \neq s\right\} .
\end{aligned}
$$




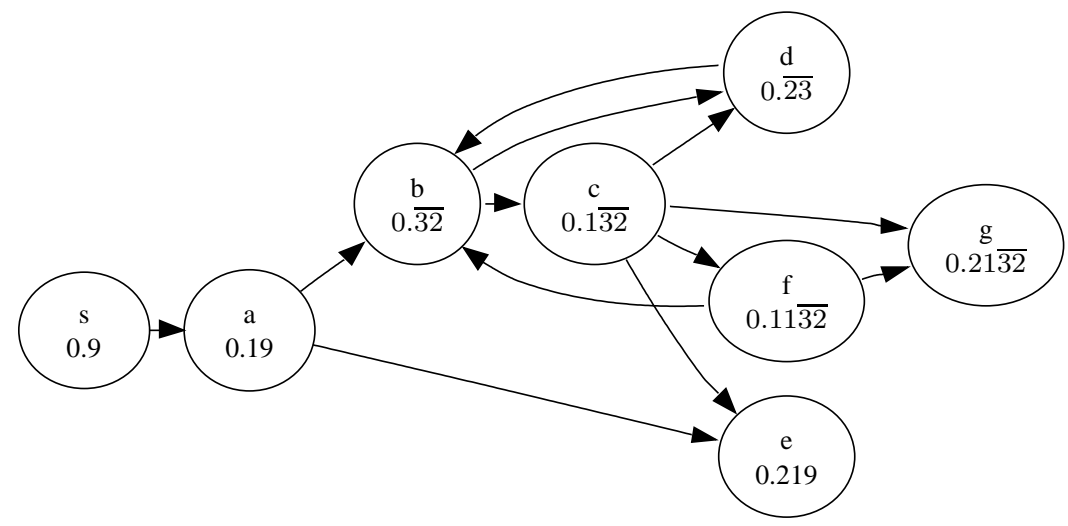

Figure 6: Values assigned by the recursive in-degree algorithm

and valuation function vp : $V^{*} \mapsto \mathbb{Q}$ is defined as:

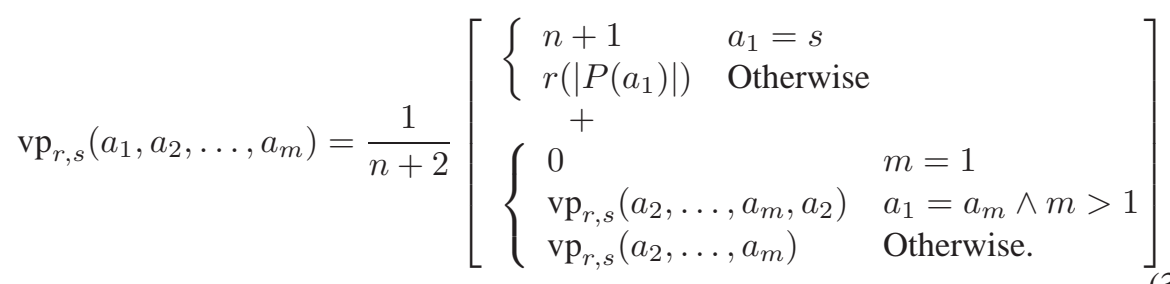

Note that $\operatorname{vp}_{r, s}\left(a_{1}, a_{2}, \ldots, a_{m}\right)$ is infinitely recursive in the case when $a_{1}=a_{m} \wedge$ $m>1$. For computation sake we can redefine this case finitely as:

$$
\begin{aligned}
\mathrm{vp}_{r, s}\left(a_{1}, \ldots, a_{m}, a_{1}\right) & =\sum_{i=0}^{\infty} \frac{1}{(n+2)^{m i}} \sum_{j=1}^{m} \frac{r\left(\left|P\left(a_{j}\right)\right|\right)}{(n+2)^{j}}= \\
& =\frac{(n+2)^{m}}{(n+2)^{m}-1} \operatorname{vp}_{r, s}\left(a_{1}, \ldots, a_{m}\right) .
\end{aligned}
$$

Further note that when the $r$ function is constant $(r \equiv 1)$, then the recursive indegree system becomes the distance system on $V_{s}$, where the vertices in $V \backslash V_{s}$ are ranked weaker, and the ordering among them is set according to the length of the longest path (simple or not) leading to the vertex.

An example of the values assigned for a particular graph when $r$ is the identity function is given in Figure 6. As $n=8$, the trust values are decimal. Note that the loop $(b, d)$ generates a periodical decimal value ${ }_{r, s}(b)=\mathrm{vp}_{r, s}(b, d)=0 . \overline{32}$ by the infinite recursion in (3).

These systems satisfy the axioms as required:

Proposition 5.7. Let $r: \mathbb{N} \mapsto \mathbb{N}$ be a monotone nondecreasing function such that $r(i) \leq i$ for all $i \in \mathbb{N}$ and define $r(0)=0$. The recursive in-degree ranking system with 
rank function $r$ satisfies self-confidence, strong quasi-transitivity and RIIA. If $r$ is not constant ${ }^{4}$ then the recursive in-degree system further does not satisfy weak maximum transitivity nor strong incentive compatibility under either $\mathcal{M}_{\text {out }}$ or $\mathcal{M}_{\text {sybil }}$.

Proof. We will prove that in the entire graph (not just $V_{s}$ ) every comparison profile $\langle\mathbf{a}, \mathbf{b}\rangle$ where $\mathbf{a}=\left(a_{1}, \ldots, a_{k}\right), \mathbf{b}=\left(b_{1}, \ldots, b_{l}\right)$ is ranked as follows:

$$
f\langle\mathbf{a}, \mathbf{b}\rangle=1 \quad \Leftrightarrow \quad(k=0) \vee(r(k)<r(l)) \vee\left[(r(k)=r(l)) \wedge\left(a_{k} \leq b_{l}\right)\right] .
$$

Note that this ranking of comparison profiles also implies strong quasi transitivity. To show comparison profiles are ranked as such, we will prove that

$\operatorname{value}_{r, s}(v)= \begin{cases}0 & v \neq s \wedge P(v)=\emptyset \\ \frac{n+1}{n+2} & v=s \\ \frac{1}{n+2}\left[r(|P(v)|)+\max _{p \in P(v)} \text { value }_{r, s}(p)\right] & \text { Otherwise }\end{cases}$

and note that $0 \leq$ value $_{r, s}(v) \leq \frac{n+1}{n+2}$, and thus vertices other than $s$ are ordered first by $r(|P(v)|)$ and then by $\max _{p \in P(v)}$ value $_{r, s}(p)$, as required. Moreover, self confidence is satisfied because for all $v \neq s$ : value r $_{r, s}(v)<\frac{n+1}{n+2}$.

The two edge cases are trivial, we shall now concentrate on the primary case in (4). Let $v \in V \backslash\{s\}$ be some vertex where $P(v) \neq \emptyset$. Denote $\operatorname{Path}_{s}^{\prime}(p, v)$ as the set of almost-simple directed paths to $p$ stopping at $s$ which do not pass through $v$ unless immediately looping back to $p$ :

$$
\begin{aligned}
\operatorname{Path}_{s}^{\prime}(p, v)=\{ & \left(p=a_{1}, a_{2}, \ldots, a_{m}\right) \mid \\
& \left(a_{m}, \ldots, a_{1}\right) \text { is a path in } G \wedge\left(a_{m-1}, \ldots, a_{1}\right) \text { is simple } \wedge \\
& \forall i \in\{1 \ldots m-1\}: a_{i} \neq s \wedge \\
& \left.\forall i \in\{1, \ldots, m-2, m\}: a_{i} \neq v \wedge a_{m-1}=v \Leftrightarrow a_{m}=p\right\} .
\end{aligned}
$$

Now we see that:

$$
\begin{aligned}
\operatorname{value}_{r, s}(v) & =\max _{\mathbf{a} \in \operatorname{Path}_{s}(v)} \operatorname{vp}_{r, s}(\mathbf{a})= \\
& =\frac{1}{n+2}\left[\begin{array}{l}
r(|P(v)|)+\max _{\left(v=a_{1}, \ldots, a_{m}\right) \in \operatorname{Path}_{s}(v)} \\
\left\{\begin{array}{ll}
\operatorname{vp}_{r, s}\left(a_{2}, \ldots, a_{m}, a_{2}\right) & a_{1}=a_{m} \wedge \\
\operatorname{vp}_{r, s}\left(a_{2}, \ldots, a_{m}\right) & \text { Otherwise. }
\end{array}\right]=1
\end{array}\right]=(5) \\
& =\frac{1}{n+2}\left[r(|P(v)|)+\max _{p \in P(v)} \max _{\mathbf{a} \in \operatorname{Path}_{s}^{\prime}(p, v)} \mathrm{vp}_{r, s}(\mathbf{a})\right]= \\
& =\frac{1}{n+2}\left[r(|P(v)|)+\max _{p \in P(v)} \max _{\mathbf{a} \in \operatorname{Path}_{s}(p)} \mathrm{vp}_{r, s}(\mathbf{a})\right]= \\
& =\frac{1}{n+2}\left[r(|P(v)|)+\max _{p \in P(v)} \operatorname{value}_{r, s}(p)\right]
\end{aligned}
$$

\footnotetext{
${ }^{4}$ If $r$ is constant, the system still does not satisfy strong incentive compatibility under either $\mathcal{M}_{\text {out }}$ or $\mathcal{M}_{\text {sybil }}$, but only if we allow vertices that have no path from $s$.
} 


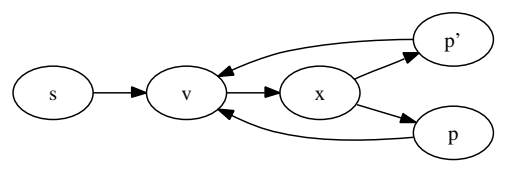

$$
\begin{aligned}
& \mathbf{a}=\left(p, x, v, p^{\prime}, x\right) \\
& \mathbf{b}=(p, x, v, p) \\
& \mathbf{c}=\left(p^{\prime}, x, v, p^{\prime}\right)
\end{aligned}
$$

Figure 7: Example of paths from the proof of Proposition 5.7.

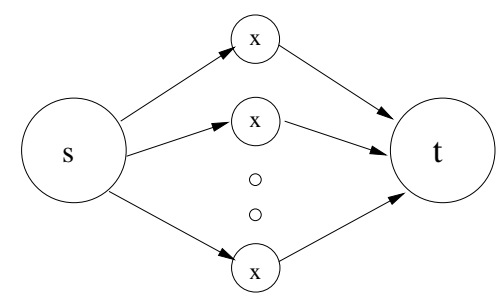

Figure 8: Graph from proof that Recursive In-degree does not satisfy axioms

To show that the equality (6) holds, assume for contradiction that there exists $p \in P(v)$ and $\mathbf{a} \in \operatorname{Path}_{s}(p)$ such that

$$
\operatorname{vp}_{r, s}(\mathbf{a})>\max _{p^{\prime} \in P(v)} \max _{\mathbf{a}^{\prime} \in \operatorname{Path}_{s}^{\prime}\left(p^{\prime}, v\right)} \operatorname{vp}_{r, s}\left(\mathbf{a}^{\prime}\right) .
$$

From $\mathbf{a} \in \operatorname{Path}_{s}(p) \backslash \operatorname{Path}_{s}^{\prime}(p, v)$, we know that $a_{i}=v$ for some $i \in\{1, \ldots, m\}$. Assume wlog that $i$ is minimal. Let $\mathbf{b}$ denote the path $\left(p=a_{1}, a_{2}, \ldots, a_{i}, p\right)$ and let c denote the path $\left(p^{\prime}=a_{i+1}, \ldots, a_{m}, a_{j+1}, \ldots, a_{i+1}\right)$ if $a_{m}=a_{j}$ for some $j<i$ or $\left(p^{\prime}=a_{i+1}, \ldots, a_{m}\right)$ otherwise. An example of such paths is given in Figure 7. Note that $\mathbf{b} \in \operatorname{Path}_{s}^{\prime}(p, v)$ and $\mathbf{c} \in \operatorname{Path}_{s}^{\prime}\left(p^{\prime}, v\right)$, where $p, p^{\prime} \in P(v)$. Now, note that

$$
\mathrm{vp}_{r, s}(\mathbf{a})=\frac{(n+2)^{j}-1}{(n+2)^{j}} \mathrm{vp}_{r, s}(\mathbf{b})+\frac{1}{(n+2)^{j}} \mathrm{vp}_{r, s}(\mathbf{c}),
$$

and thus $\mathrm{vp}_{r, s}(\mathbf{a})$ must be between $\mathrm{vp}_{r, s}(\mathbf{b})$ and $\mathrm{vp}_{r, s}(\mathbf{c})$, in contradiction to assumption (7).

We shall now prove that recursive in-degree is not incentive compatible under $\mathcal{M}_{\text {out }}$ or $\mathcal{M}_{\text {sybil }}$ and does not satisfy weak maximum transitivity. Let $i \in \mathbb{N}$ be the minimum number such that $r(i)>1$. Consider the graph $G$ in Figure 8, where there are $i$ vertices labeled $x$. This graph is ranked $x \prec t \prec s$, where $x$ refers to all vertices labeled $x$. Weak maximum transitivity is not satisfied because $x \prec t$ even though $s \succ x$. Let $x^{\prime}$ be one of the vertices labeled $x$. It can perform a manipulation in $\mathcal{M}_{\text {out }}$ by removing its edge to $t$, and thus changing the ranking to $x \simeq x^{\prime} \simeq t \prec s$. It can also perform a manipulation in $\mathcal{M}_{\text {sybil }}$ by creating $i$ additional sybils of themselves and create a complete clique thus changing the ranking to $x \prec v \simeq x^{\prime} \simeq t \prec s$, where $v$ are the new vertices involved from the manipulation. 
For an extensive study of the recursive in-degree system in the context of general ranking systems see [6].

\section{Concluding Remarks}

We have presented a method for the evaluation of personalized ranking systems by using axioms adapted from the ranking systems literature, and evaluated existing and new personalized ranking systems according to these axioms. As most existing PRSs do not satisfy these axioms, we have presented several new and practical personalized ranking systems that satisfy subsets, or indeed all, of these axioms. We argue that these new ranking systems have a more solid theoretical basis, and thus may very well be successful in practice.

Furthermore, we have proven a representation theorem for the Strong Count ranking systems, which are the only systems that satisfy all axioms.

This study is far from exhaustive. Further research is due in formulating new axioms, and proving representation theorems for the various PRSs suggested in this paper. An additional avenue for research is modifying the setting in order to accommodate for more elaborate input such as trust/distrust relations or numerical trust ratings, as seen in some existing personalized ranking systems used in practice.

\section{References}

[1] Alon Altman and Moshe Tennenholtz. On the axiomatic foundations of ranking systems. In Proc. 19th International Joint Conference on Artificial Intelligence, pages 917-922, 2005.

[2] Alon Altman and Moshe Tennenholtz. Ranking systems: the pagerank axioms. In $E C$ ' 05 : Proceedings of the 6th ACM conference on Electronic commerce, pages 1-8, New York, NY, USA, 2005. ACM Press.

[3] Alon Altman and Moshe Tennenholtz. An axiomatic approach to personalized ranking systems. In Proc. 20th International Joint Conference on Artificial Intelligence, 2006.

[4] Alon Altman and Moshe Tennenholtz. Incentive compatible ranking systems, 2006. Technical report, Technion.

[5] Alon Altman and Moshe Tennenholtz. Quantifying incentive compatibility of ranking systems. In Proc. of AAAI-06, 2006.

[6] Alon Altman and Moshe Tennenholtz. Axiomatic foundations for ranking systems. Technical Report, Technion, 2007.

[7] K.J. Arrow. Social Choice and Individual Values (2nd Ed.). Yale University Press, 1963.

[8] Paolo Avesani, Paolo Massa, and Roberto Tiella. A trust-enhanced recommender system application: Moleskiing. In SAC '05: Proceedings of the 2005 ACM symposium on Applied computing, pages 1589-1593, New York, NY, USA, 2005. ACM Press.

[9] Allan Borodin, Gareth O. Roberts, Jeffrey S. Rosenthal, and Panayiotis Tsaparas. Link analysis ranking: algorithms, theory, and experiments. ACM Trans. Inter. Tech., 5(1):231297, 2005.

[10] J. Callas, L. Donnerhacke, H. Finney, and R. Thayer. RFC 2440: OpenPGP message format, November 1998. Status: PROPOSED STANDARD. 
[11] Alice Cheng and Eric Friedman. Sybilproof reputation mechanisms. In P2PECON '05: Proceeding of the 2005 ACM SIGCOMM workshop on Economics of peer-to-peer systems, pages 128-132, New York, NY, USA, 2005. ACM Press.

[12] Bhaskar Dutta, Matthew O Jackson, and Michel Le Breton. Strategic candidacy and voting procedures. Econometrica, 69(4):1013-37, 2001.

[13] A. Gibbard. Manipulation of voting schemes. Econometrica, 41:587-601, 1973.

[14] T. Haveliwala, S. Kamvar, and G. Jeh. An analytical comparison of approaches to personalizing pagerank, 2003. Technical report, Stanford University.

[15] Jon M. Kleinberg. Authoritative sources in a hyperlinked environment. Journal of the ACM (JACM), 46(5):604-632, 1999.

[16] Raph Levien. Attack Resistant Trust Metrics. PhD thesis, University of California, Berkeley, 2002.

[17] Paolo Massa and Paolo Avesani. Controversial users demand local trust metrics: An experimental study on epinions.com community. In Proc. of AAAI-05, pages 121-126, 2005.

[18] L. Page, S. Brin, R. Motwani, and T. Winograd. The pagerank citation ranking: Bringing order to the web. Technical Report, Stanford University, 1998.

[19] I. Palacios-Huerta and O. Volij. The measurement of intellectual influence. Econometrica, 73(3), 2004.

[20] P. Resnick and R. Zeckhauser. Trust among strangers in internet transactions: Empirical analysis of ebay's reputation system. Working Paper for the NBER workshop on empirical studies of electronic commerce, 2001.

[21] M.A. Satterthwaite. Stratey proofness and arrow's conditions: Existence and correspondence theorems for voting procedures and social welfare functions. Journal of Economic Theory, 10:187-217, 1975.

[22] M. Tennenholtz. Reputation systems: An axiomatic approach. In Proceedings of the 20th conference on uncertainity in Artificial Intelligence (UAI-04), 2004. 